\title{
Healthcare burden of rare diseases in Hong Kong - adopting ORPHAcodes in ICD-10 based healthcare administrative datasets
}

\author{
Annie Ting Gee Chiu', Claudia Ching Yan Chung², Wilfred Hing Sang Wong ${ }^{2}$, So Lun Lee² \\ and Brian Hon Yin Chung ${ }^{2^{*}}$ (D)
}

\begin{abstract}
Background: The burden of rare diseases is important for healthcare planning but difficult to estimate. This has been facilitated by the development of ORPHAcodes, a comprehensive classification and coding system for rare diseases developed by the international consortium Orphanet, with cross-references to the 10th version of the International Classification of Diseases and Related Health Problems (ICD-10). A recent study in Western Australia made use of this cross-referencing to identify rare diseases-related admissions in health administrative datasets. Such methodology was adopted in Hong Kong, which has a population of 7 million comprising of 92\% ethnic Chinese, with over $80 \%$ of admissions taking place in the public hospitals and available for review from the local public healthcare database.
\end{abstract}

Main body: Our objective was to identify the inpatient healthcare burden of rare diseases in Hong Kong. We extracted admission records of all patients coded with one or more of the 1084 ICD-10 codes cross referenced with 467 ORPHAcodes during the study period from 1st January 2005 to 31st December 2016. We further analysed rare disease-related inpatient healthcare cost using a subset of patients admitted during 1st April 2015 - 31st March 2016. A total number of 546,673 admissions were identified, representing 3.2\% of total admissions during the study period. By the end of the study, 109,535 patients were alive, representing 1.5\% of the overall population. Prevalence of rare diseases was found to be 1 in 67 in the Hong Kong population. The most common rare disease category in the paediatric age group was 'rare developmental defect during embryogenesis'; whereas that amongst adults was 'rare haematologic disease'. The aforementioned subset of patients accounted for 330,091 inpatient-days, placing the estimated total inpatient cost for rare disease population at $\operatorname{HKD} \$ 1,594,339,530$ i.e. 4.3\% of total inpatient cost in 2015-2016.

Conclusion: Cross referencing between ICD-10 and ORPHAcodes may be adopted in different healthcare datasets for international comparison. Despite differences in the prevalence of individual disease, the disparity between rare disease prevalence (1.5\%) and associated inpatient cost (4.3\%) in Hong Kong reflects the importance of rare diseases in healthcare policies.

Keywords: Rare diseases, ORPHAcodes, ICD-10, Disease burden, Prevalence, Inpatient cost, Hong Kong

\footnotetext{
* Correspondence: bhychung@hku.hk

${ }^{2}$ Department of Paediatrics and Adolescent Medicine, The University of Hong

Kong, Hong Kong, SAR, People's Republic of China

Full list of author information is available at the end of the article
}

(c) The Author(s). 2018 Open Access This article is distributed under the terms of the Creative Commons Attribution 4.0 International License (http://creativecommons.org/licenses/by/4.0/), which permits unrestricted use, distribution, and reproduction in any medium, provided you give appropriate credit to the original author(s) and the source, provide a link to the Creative Commons license, and indicate if changes were made. The Creative Commons Public Domain Dedication waiver (http://creativecommons.org/publicdomain/zero/1.0/) applies to the data made available in this article, unless otherwise stated. 


\section{Background}

Rare diseases refer to conditions with rare occurrences in a population. They are often life threatening or chronically debilitating [1], but with limited treatment owing to lack of incentive in marketing (Rare Disease Act of 2002, United States). The number of rare diseases is estimated at 5000 to 8000 . They are individually rare but collectively affect $6-8 \%$ of the European population [2] i.e. similar to the proportion of people living with asthma or diabetes [3].

The disease burden of rare diseases may be gauged either from the individual's perspective or from its overall impact on the healthcare system. From the individual patient's perspective, $25 \%$ of patients had to wait 5 to 30 years from disease onset to receiving a diagnosis; and in $40 \%$ the initial diagnosis was wrong, according to EURORDIS, a rare disease patient organization in Europe [4]. A similar study performed in Australia showed that $30 \%$ of the patients waited more than 5 years to receive a diagnosis, $60 \%$ saw more than 3 doctors before receiving a diagnosis and nearly half of the patients received at least one incorrect diagnosis during their diagnostic odyssey [5].

The impact of rare diseases on the healthcare system is harder to gauge. Firstly, there is a lack of universal definition for rare disease, with prevalence threshold varying across countries, ranging from 5 to 76/ 100,000 and an average threshold of 40/100,000 people [6]. Secondly, new diagnoses are fast emerging, with discovery of more than 100 new diseases per year from 2010 to 2015 [7]. As such there have only been a few studies on the healthcare burden of rare diseases, which mostly utilize national registries for rare diseases or national disability registry for estimation $[8,9]$. They are helpful in delineating rare disease related demographics but rely on reporting from individual practitioners and cannot reflect more complicated information such as inpatient attendance or length of stay.

Currently a few rare disease nosologies exist, the most common ones being the Orphanet Rare Disease Ontology (ORDO) and Online Mendelian Inheritance in Man (OMIM). Whereas OMIM is mostly used in genetic databases, ORDO is a structured vocabulary for rare diseases derived from the orphanet database, which defines rare disease as occurring in less than 1 in 2000. It captures relationship between diseases, genes and other relevant features to form a useful resource for computational analysis of rare diseases, and has connections with other terminologies or classification system such as the 10th version of the International Classification of Diseases and Related Health Problems (ICD-10). Whereas ICD-10 contains only 500 unique codes for rare diseases, it is now overcome by the cross referencing with ORPHAcodes, a comprehensive classification and coding system for rare diseases developed by the international consortium
Orphanet [10]. ORPHAcodes are now increasingly used by European healthcare systems for informatics tracing of rare diseases, and their introduction is fostered by National Action Plans and Strategies for Rare Diseases and recommended by the European Commission expert group on rare diseases.

A recent study by Walker et al. made use of health administrative data sets to identify admissions related to rare diseases in Western Australia [11]. This was achieved through cross referencing between ORPHAcodes and an Australian modification of ICD-10 (ICD-10-AM). Using back-and-forth translation, a panel of medical experts came up with 1084 disease codes and used this as the basis for calculation of healthcare burden relating to rare disease. This study was a paradigm shift that allowed for not only the calculation of prevalence but also that of healthcare cost relating to rare diseases [11]. It also provided an opportunity for international comparison using comparable methodologies.

Hong Kong has a population of 7 million with approximately $92 \%$ of the population being ethnic Chinese [12]. More than $80 \%$ of admissions take place in the public hospitals under the management of the Hospital Authority (HA) [13]. Within HA, all inpatient diagnosis are recorded using ICD-10, all of which are available in the Clinical Data Analysis and Reporting System (CDARS) in an unlisted and anonymous manner for research purposes.

Rare disease has recently gained more public awareness in Hong Kong, as evident by both the establishment of the Hong Kong Alliance of Rare Disease in 2014 and legislative council debates on rare disease in 2016-2017. The recent spotlight on rare diseases highlighted the lack of local epidemiological studies on rare diseases. To bridge this literature gap, we adopted the methodology described by Walker et al. [11] in the public healthcare administrative system of Hong Kong. In this study, we sought to i) estimate the prevalence and distribution of rare diseases in Hong Kong; ii) ascertain the number and proportion of admissions in rare diseases requiring inpatient care; and iii) estimate the inpatient-related healthcare cost in patients with rare diseases in Hong Kong.

\section{Methods}

\section{Rare disease diagnostic coding}

The Hospital Authority of Hong Kong adopts the Hong Kong Clinical Terminology Table (HKCTT) for diagnostic coding purposes. The HKCTT contains mapped codes to three versions of ICD-10, namely ICD-10 2001, ICD-10 2010, and ICD-10 Mental Health and Behavioural Disorders (ICD-10 MBD), which are searchable on the CDARS database [14] and reported to be reliable in previous epidemiological studies $[15,16]$.

We adopted the 1084 ICD-10-AM codes set out by Walker et al. [11] in our study, which were matched to 
467 ORPHAcodes with back-and-forth translation and verified using period prevalence with exclusion of infectious diseases. Records corresponding to the above diagnostic coding were identified from the CDARS database.

\section{Study population}

This retrospective study included all patients who had an inpatient admission at one or more of the hospitals under the HA with one or more of the above 1084 diagnostic codes between 1st January 2005 and 31st December 2016. Other extracted variables included the patients' age at admission, gender, length of stay, and mortality (as defined by death recorded in the Hong Kong Death Registry).

\section{Data analysis}

Demographic information about the study population and their admissions to public hospitals in Hong Kong, including the number of subjects, number of admissions, male to female ratio and age distribution were calculated.

Rare diseases were analysed by their ORPHAcode categories. All patients in the CDARS database were recorded in an unlisted and anonymous manner by a unique reference key (CDARS ID number, which is different from the Hong Kong Identity Card number). Multiple lines of entries with the same reference key were found when the same patient has been admitted into any of the hospitals under the HA system for more than once. The total number of admissions, total number of rare disease patients, and the number of patients in each disease category were calculated. Multiple admissions with one of the 1084 diagnostic codes for the same patient were included when considering the total number of admissions; whereas the reference keys were sorted and counted once only by their unique codes using Microsoft Excel when considering the total number of patients in the study and the number of patients in each disease category.

Patient's age at admission was used for the paediatric and adult subgroup analysis, this could be calculated using the date of admission and the date of birth for each patient, which were recorded in the CDARS database. When considering the number of patients in the paediatric and adult population, it was presumed that some patients would have had admissions both before and after their 18th birthday, the age of the patient's first admission was used because it was known that some rare diseases have an earlier onset than others.

The average, median, and interquartile range (25\% and $75 \%$ quartile) of the length of stay in each disease category were also calculated. Instead of using the $95 \%$ confidence interval which assumed the data to follow a normal distribution, the interquartile range was used as the dataset was skewed by some patients with a very prolonged LOS.

The surviving number of patients on 31st December 2016 was identified for calculation of overall and category specific rare disease prevalence, using the Hong Kong Census data from the end of 2016 [12]. A subset of data between 1st April 2015 and 31st March 2016 (representing a fiscal year for the HA) was further identified. Using this set of data, the overall cost of inpatient stay for rare disease patients was calculated, which was assumed to be the same over the study period, with a unit cost of HKD\$4830 (i.e. USD\$619) per inpatient-day (acute and convalescent beds) according to the HA 2015-2016 data [13]. This cost was an actual mean cost calculated in the general population (including rare diseases patients) in 2015-2016. It was understood that the cost per inpatient-day would be highly variable among different diseases treated, however, disease-specific inpatient cost data is not available in the local setting.

Data analysis was performed using $\mathrm{R}$ (version 3.4.1).

\section{Results}

\section{Whole cohort}

A total of 144,444 patients were identified from the CDARS database, with male to female ratio of 1:1.05. There were 546,673 admissions, with the paediatric to adult admission ratio being 1:3.09 (see Table 1). The under 5 years age group had the largest total number of admissions, accounting for $17.8 \%$ of the total number of admissions in this cohort (see Table 2), and $7.1 \%$ of the under 5 years age group in the general population over the study period.

The majority (95\%) of the cohort had only one rare disease diagnosis pertaining to one rare disease category; 27,075 (5.0\%) had rare disease diagnoses from two or more rare disease category. These patients were included in the calculations of all the respective disease categories but only counted once in the overall counts for patients and admissions. Over the study period, the rare disease category with the most patients was 'rare developmental defect during embryogenesis'; whereas the rare disease category with the highest number of inpatient admissions (including repeated admissions) was 'rare haematologic disease'. Within the paediatric age group, 'rare developmental defect during embryogenesis' was the most common rare disease category, whereas 'rare haematologic disease' was the most common rare disease category within the adult age group (see Table 3).

A total of 34,909 deaths were recorded over the study period (96.8\% contributed by the adult population); 109,535 were alive (75.8\%) as of 31 December 2016. Consequently, in December 2016 the whole rare disease population represented $1.5 \%$ of the Hong Kong population. Within the paediatric age group, 'rare hepatic disease' had the highest proportion of mortality (25.4\%), 
Table 1 Basic demographics of patients with rare diseases (12-year cohort from 2005 to 2016)

\begin{tabular}{ll}
\hline Study period & $01-01-2005$ to 31-12-2016 \\
Total population & 144,444 \\
Total number of admissions & 546,673 \\
Male: female ratio & $1: 1.05$ \\
$\begin{array}{l}\text { Paediatric ( } \leq 18 \text { years): adult admission } \\
\text { (>18 years) }\end{array}$ & $1: 3.09$ \\
\hline
\end{tabular}

whereas 'rare neoplastic disease' had the highest proportion of mortality within the adult age group (74.4\%). The relative proportion of rare diseases and mortality of different rare disease categories in paediatric and adult age groups is represented in Fig. 1.

The average and median length of stay (LOS) were 6.7 days and 1 day respectively in the rare disease population. Within the under-five age group, the average and median LOS were 8.7 days and 1 day respectively.

'Rare respiratory disease' has the longest average length of stay, followed by 'rare eye disease'. It should be noted that the median LOS are considerably shorter than that of the average LOS across all disease categories, suggesting that the dataset is skewed towards shorter LOS with some patients having very prolonged LOS (see Table 4).

\section{5-2016 cohort}

Further analysis was performed using the 1st April 2015 - 31st March 2016 cohort, which included 22,606 patients and 54,488 admissions. The mean length of stay was 6.1 days, which was marginally higher than that of the general population (5.8 days). The period prevalence of rare disease was estimated at 1 in 67 people using the Hong Kong Census data from the end of 2016 [12].

There were a total of 330,091 inpatient-days in the above period. Taking the unit cost per inpatient-day provided by the HA for the year 2015-2016, which was HKD\$4830 (i.e. USD\$619) per day, the estimated total inpatient cost was HKD $\$ 1,594,339,530$ (i.e. USD $\$ 204,402,504$ ), accounting for $4.3 \%$ of the total inpatient cost for all diseases in Hong Kong, in which the total inpatient cost for all diseases was estimated at $\mathrm{HKD} \$ 37,467,184,230$ (i.e. USD $\$ 4,803,485,158$ ) (see Table 5).

\section{Discussion}

The current study analysed the inpatient burden of healthcare from patients with rare disease. The 467 studied rare diseases represented $1.5 \%$ of the Hong Kong population by 2016 , and accounted for approximately $4.3 \%$ of the inpatient hospital costs for that year. This disparity does not only contribute to the immediate healthcare cost, but will also have substantial social and economic implications for patients, family members, and carers [17].

Table 2 Distribution of rare diseases related hospital admissions by age at admission in Hong Kong (2005-2016)

\begin{tabular}{|c|c|c|}
\hline Age group & $\begin{array}{l}\text { Number of admissions in the rare disease population } \\
\text { (\% of the total rare disease population) }\end{array}$ & $\begin{array}{l}\text { Number of admissions in the general population (\% of the } \\
\text { total general population, including all diseases) }\end{array}$ \\
\hline $0-4$ & $97,416(17.8 \%)$ & $1,373,714(8.0 \%)$ \\
\hline $5-9$ & 34,438 (6.3\%) & $259,111(1.5 \%)$ \\
\hline $10-14$ & 26,776 (4.9\%) & $208,713(1.2 \%)$ \\
\hline $15-19$ & $28,565(5.2 \%)$ & $256,811(1.5 \%)$ \\
\hline $20-24$ & 26,808 (4.9\%) & 369,707 (2.2\%) \\
\hline $25-29$ & 27,598 (5.0\%) & $597,883(3.5 \%)$ \\
\hline $30-34$ & 28,001 (5.1\%) & 824,150 (4.8\%) \\
\hline $35-39$ & 21,554 (3.9\%) & 768,238 (4.5\%) \\
\hline $40-44$ & 17,983 (3.3\%) & 709,462 (4.2\%) \\
\hline $45-49$ & 21,336 (3.9\%) & $914,342(5.3 \%)$ \\
\hline $50-54$ & 28,092 (5.1\%) & $1,161,757$ (6.8\%) \\
\hline $55-59$ & 30,965 (5.7\%) & $1,284,643(7.5 \%)$ \\
\hline $60-64$ & $30,686(5.6 \%)$ & $1,273,321(7.5 \%)$ \\
\hline $65-69$ & $28,174(5.2 \%)$ & $1,238,402(7.2 \%)$ \\
\hline $70-74$ & $25,249(4.6 \%)$ & $1,298,569(7.6 \%)$ \\
\hline $75-79$ & 27,861 (5.1\%) & $1,494,884(8.7 \%)$ \\
\hline $80-84$ & 23,493 (4.3\%) & $1,397,762(8.2 \%)$ \\
\hline $85+$ & $21,678(4.0 \%)$ & $1,659,092(9.7 \%)$ \\
\hline Total & 546,673 & $17,090,561$ \\
\hline
\end{tabular}


Table 3 Rare disease in Hong Kong at a glance (2005-2016)

\begin{tabular}{|c|c|c|c|c|}
\hline Orphanet classification & Number of people (\%) & Mean (median) age & $\begin{array}{l}\text { Number of people } \\
<=18 \text { years old (\%) }\end{array}$ & $\begin{array}{l}\text { Estimated prevalence, } \\
\text { as of } 2016\end{array}$ \\
\hline Rare developmental defect during embryogenesis disease & $33,731(21.9)$ & $21.4(11.9)$ & $23,835(70.7)$ & 457 per 100,000 \\
\hline Rare haematologic disease & $24,615(16.0)$ & $51.8(37)$ & $3331(13.5)$ & 334 per 100,000 \\
\hline Rare neurologic disease & $23,707(15.4)$ & $56.1(57.7)$ & $4507(19.0)$ & 321 per 100,000 \\
\hline Rare neoplastic disease & $14,556(9.4)$ & $70.6(61.0)$ & $757(5.2)$ & 197 per 100,000 \\
\hline Rare endocrine disease & $9923(6.4)$ & $48.9(53.7)$ & $2893(29.2)$ & 135 per 100,000 \\
\hline Rare systemic or rheumatologic disease & $8678(5.6)$ & $42.0(48.5)$ & $3411(39.3)$ & 118 per 100,000 \\
\hline Rare otorhinolaryngologic disease & $7726(5.0)$ & $54.4(60.0)$ & $2052(26.6)$ & 105 per 100,000 \\
\hline Rare cardiac disease & $6581(4.3)$ & $63.9(65.0)$ & $300(4.6)$ & 89 per 100,000 \\
\hline Rare respiratory disease & $4810(3.1)$ & $21.4(10.5)$ & $3739(77.7)$ & 65 per 100,000 \\
\hline Rare inborn errors of metabolism & $4287(2.8)$ & $57.1(44.6)$ & $707(16.5)$ & 58 per 100,000 \\
\hline Rare eye disease & $3303(2.1)$ & $22.6(9.9)$ & $2422(73.3)$ & 45 per 100,000 \\
\hline Rare skin disease & $2646(1.7)$ & $68.1(79.8)$ & $220(8.3)$ & 36 per 100,000 \\
\hline Rare renal disease & $2532(1.6)$ & $12.9(8.7)$ & $2230(88.1)$ & 34 per 100,000 \\
\hline Rare hepatic disease & $2455(1.6)$ & $69.8(71.0)$ & $66(2.7)$ & 33 per 100,000 \\
\hline Rare gastroenterologic disease & $2447(1.6)$ & $72.0(78.8)$ & $24(1.0)$ & 33 per 100,000 \\
\hline Rare bone disease & $1191(0.8)$ & $39.9(37.0)$ & $408(34.3)$ & 16 per 100,000 \\
\hline Rare gynecologic or obstetric disease & $653(0.4)$ & $48.5(46.0)$ & $2(0.3)$ & 9 per 100,000 \\
\hline Rare urogenital disease & $119(0.1)$ & $39.3(34.1)$ & $27(22.7)$ & 2 per 100,000 \\
\hline Rare immune disease & $78(0.1)$ & $30.2(35.7)$ & $45(57.7)$ & 1 per 100,000 \\
\hline Rare odontologic disease & $32(0.0)$ & $40.4(45.2)$ & $11(34.4)$ & $<1$ per 100,000 \\
\hline Rare abdominal surgical disease & $5(0.0)$ & $56.1(57.3)$ & $0(0.0)$ & 1 per $1,000,000$ \\
\hline Rare circulatory system disease & $2(0.0)$ & $36.6(36.6)$ & $0(0.0)$ & $<1$ per $1,000,000$ \\
\hline Total & 144,444 & & & \\
\hline
\end{tabular}

The utilisation of CDARS allowed identification of all patient data in Hong Kong under the HA, which handles more than $80 \%$ of all admission in Hong Kong. CDARS is proven to be a reliable source of health administrative data [18], and the adoption of the methodology by Walker et al. in CDARS allowed international comparison of rare disease related epidemiological data. Both studies showed that the inpatient cost of rare diseases is disproportionally higher than its prevalence. Likewise, rare developmental defect during embryogenesis, which accounted for nearly one-third of the list of ORPHAcodes, has the highest patient load in both this study and the Western Australia cohort [11].

There were however, some notable difference in disease distribution between the two cohorts. In the Western Australian cohort, rare neoplastic diseases had the highest adult prevalence, whereas in the current cohort, this was superseded by rare haematologic diseases. We postulate that such difference could be attributed to the difference in disease burden across ethnic groups, eg. higher prevalence of thalassaemia major, lower prevalence of rare neoplasms such as Hodgkin lymphoma in ethnic Chinese, which is the predominant in Hong Kong. We also note a difference in disease burden between the paediatric and adult population, the most significant one being the predominance of rare developmental defect during embryogenesis disease (48.2\%) in the paediatric population, which only constitute $11.2 \%$ of rare diseases in the adult population. On the other hand, the proportion of patients with rare haematologic diseases increases from $6.1 \%$ in the paediatric rare disease population to $20 \%$ in adulthood. This increment is matched by rare neoplastic diseases (from 1.3\% in paediatric age group to $12.8 \%$ in adulthood) and rare neurologic diseases (from $7 \%$ in paediatric age group to $18 \%$ in the adulthood). Of interest is that rare inborn errors of metabolism, which is traditionally thought to predominate in childhood, is actually more prevalent in the adult rare disease population (3.4\%) than in children (1.2\%).

Whilst cross referencing between ORPHAcodes and ICD-10 in health administrative datasets facilitated international comparison and provided an overview of rare disease burden, it did not allow for in-depth analysis. The estimation of $1.5 \%$ of the Hong Kong population is noticeably lower than the reported estimates of $2.0 \%$ in the Western Australia study [11], and 6-8\% from the 


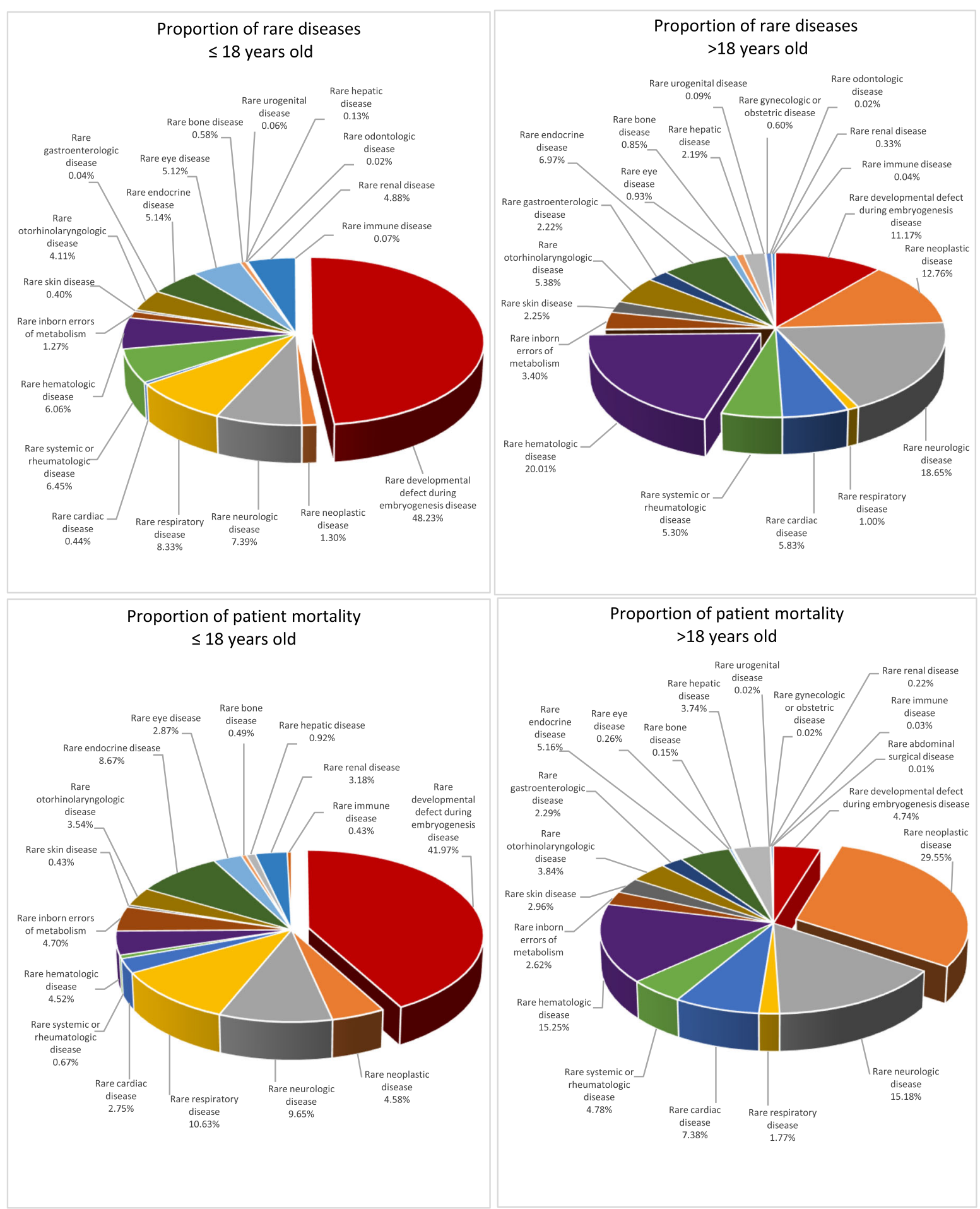

Fig. 1 Proportion of rare diseases and mortality in paediatric and adult age patients from various rare disease categories

European Commission estimates [2]. Since the CDARS system is not linked to the Hong Kong census data, results from this study may be an underestimate of the number of people living with rare diseases and should be considered as minimum values. Patients who have never used any services under the HA system, or those who have used other services such as outpatient, accident and emergency, and allied health services without the use of inpatient services were not included in this study. Total inpatient cost in this study was also likely 
Table 4 Discharges and LOS of inpatient healthcare amongst people with rare diseases in Hong Kong (2005-2016)

\begin{tabular}{|c|c|c|c|c|}
\hline Cohort & $\begin{array}{l}\text { Total admission over } \\
\text { the study period }\end{array}$ & $\begin{array}{l}\text { Total hospital } \\
\text { stay in days }\end{array}$ & Mean (Median) LOS & $25-75 \%$ quartile LOS \\
\hline All discharges & 546,673 & $3,635,917$ & $6.7(1)$ & $1-4$ \\
\hline Rare haematologic disease & 135,503 & 418,635 & $3.1(1)$ & $1-149$ \\
\hline Rare neoplastic disease & 121,824 & 691,149 & $5.7(1)$ & $1-157$ \\
\hline $\begin{array}{l}\text { Rare developmental defect during } \\
\text { embryogenesis disease }\end{array}$ & 99,638 & 736,445 & $7.4(1)$ & $1-618$ \\
\hline Rare neurologic disease & 54,954 & 775,579 & $14.1(3)$ & $1-954$ \\
\hline Rare systemic or rheumatologic disease & 31,408 & 186,689 & $5.9(1)$ & $1-115$ \\
\hline Rare endocrine disease & 26,518 & 187,198 & $7.1(1)$ & $1-201$ \\
\hline Rare cardiac disease & 22,944 & 153,627 & $6.7(3)$ & $1-92$ \\
\hline Rare inborn errors of metabolism & 22,483 & 95,734 & $4.3(1)$ & $1-121$ \\
\hline Rare otorhinolaryngologic disease & 13,044 & 116,342 & $8.9(2)$ & $1-183$ \\
\hline Rare respiratory disease & 9345 & 284,592 & $30.5(7)$ & $1-290$ \\
\hline Rare hepatic disease & 6436 & 57,788 & $9.0(4)$ & $1-69$ \\
\hline Rare gastroenterologic disease & 5804 & 26,699 & $4.6(2)$ & $1-40$ \\
\hline Rare eye disease & 5709 & 141,814 & $24.8(1)$ & $1-195$ \\
\hline Rare renal disease & 5539 & 74,345 & $7.9(2)$ & $1-77$ \\
\hline Rare skin disease & 4811 & 67,255 & $14.0(5)$ & $1-95$ \\
\hline Rare bone disease & 2873 & 38,580 & $13.4(2)$ & $1-88$ \\
\hline Rare immune disease & 2219 & 5963 & $2.7(1)$ & $1-9$ \\
\hline Rare gynecologic or obstetric disease & 826 & 1156 & $1.4(1)$ & $1-2$ \\
\hline Rare urogenital disease & 259 & 713 & $2.8(1)$ & $1-2$ \\
\hline Rare odontologic disease & 33 & 46 & $1.4(1)$ & $N A^{a}$ \\
\hline Rare abdominal surgical disease & 6 & 27 & $4.5(3)$ & $N A^{a}$ \\
\hline Rare circulatory system disease & 4 & 26 & $6.5(2)$ & $N A^{a}$ \\
\hline
\end{tabular}

LOS length of stay

${ }^{a}$ NA due to limited observations for calculation

underestimated owing to the unavailability of disease-specific inpatient cost listing in Hong Kong, and was calculated instead using the average inpatient cost within the HA. This included the clinical, biochemical and pathology investigation and general nursing fees only, and did not include other important costs such as pharmaceutical, inpatient allied health services and equipment costs. Moreover, the ORPHAcodes used in this study were only matched to 467 of the estimated 6000 to 8000 rare diseases [11], which further contributes to the underestimation of the true prevalence of rare disease. The overall rare disease prevalence remains unknown. Total healthcare cost attributed to rare disease is expected to be significantly higher with the inclusion of other healthcare services. In addition, disease-specific inpatient cost data is not available in the local setting, and that medication cost has not yet been taken into account in the current calculations, thus the true disparity between prevalence and proportion of healthcare cost is likely much higher.

\section{Conclusion}

Despite its limitations, the current study adds to available literature by demonstrating the feasibility of

Table 5 Total number of inpatients, admissions, inpatient-days and total inpatient costs of rare disease population (2015-2016)

\begin{tabular}{llll}
\hline & $\begin{array}{l}\text { Total number } \\
\text { of inpatients }\end{array}$ & $\begin{array}{l}\text { Total number } \\
\text { of admission }\end{array}$ & $\begin{array}{c}\text { Total inpatient-days inpatient } \\
\text { cost (HKD) }\end{array}$ \\
\hline $\begin{array}{l}2015-2016 \text { rare disease population } \\
\text { (\% as compared to the general population) }\end{array}$ & $22,606(2.9 \%)$ & $54,488(5.06 \%)$ & 330,091 \\
$\begin{array}{l}\text { General population (all diseases including } \\
\text { rare diseases) }\end{array}$ & 777,616 & $1,077,325$ & $\mathbf{7 , 7 5 7 , 1 8 1}$ \\
\hline
\end{tabular}


adopting ORPHAcodes in the estimation of rare disease burden in Hong Kong. It also demonstrates the comparability of inpatient rare disease burden across different medical systems and helps to delineate rare disease distribution across ages. Subgroup analyses between the paediatric and adult population further provides important information for service planning in Hong Kong, such as the opening of Hong Kong Children Hospital in 2018.

Future areas for research include the extension of rare disease related healthcare cost calculation to intensive care, outpatient and medication related costs. The societal impact should also be considered by incorporating carer cost, production cost and trade-offs etc. However, costs and trade-offs associated with children is hard to measure, thus future research should also involve the development of instruments to capture these aspects. As public awareness for rare disease patients increases, it will be important to delineate the overlap between terms such as rare disease, children with medical complexities, children requiring palliative care, which are sometimes mistakenly or intentionally used interchangeably. As medical care for rare diseases advances and the children with once incurable conditions now live into and well beyond adulthood, further studies on transitional care will also be important.

\section{Abbreviations \\ CDARS: Clinical Data Analysis and Reporting System; HA: Hospital Authority; HKCTT: Hong Kong Clinical Terminology Table; ICD-10: the 10th version of the International Classification of Disease and Related Health Problems; ICD- 10-AM: Australian modification of the 10th version of the International Classification of Disease and Related Health Problems; OMIM: Online Mendelian Inheritance in Man; ORDO: Orphanet Rare Disease Ontology}

\section{Acknowledgements}

We would like to acknowledge the Society for the Relief of Disabled Children (Hong Kong) for their financial support.

\section{Funding}

The authors would like to acknowledge and thank the Society for the Relief of Disabled Children (Hong Kong) for funding support.

\section{Availability of data and materials}

The data that support the findings of this study are available from the Hong Kong Hospital Authority but restrictions apply to the availability of these data, which were used under license for the current study, and so are not publicly available. Data are however available from the authors upon reasonable request and with permission of the Hong Kong Hospital Authority.

\section{Authors' contributions}

ATGC, CCYC, WHSW, and BHYC involved in the study design and project execution. ATGC and CCYC drafted the manuscript. CCYC and WHSW involved in data collection, data analysis and data organisation and presentation. WHSW, SLL and BHYC critically reviewed the manuscript with suggestions for improvement and revision. SLL and BHYC were the principal investigators of the project. All of the authors reviewed and approved the final manuscript.

\section{Ethics approval and consent to participate}

The study was approved by the Institutional Review Board of the University of Hong Kong/ Hospital Authority Hong Kong West Cluster (IRB reference number: UW 13-323).
Consent for publication

Not applicable

\section{Competing interests}

The authors declare that they have no competing interests.

\section{Publisher's Note}

Springer Nature remains neutral with regard to jurisdictional claims in published maps and institutional affiliations.

\section{Author details}

'Department of Paediatrics and Adolescent Medicine, Queen Mary Hospital, Hong Kong, SAR, People's Republic of China. ${ }^{2}$ Department of Paediatrics and Adolescent Medicine, The University of Hong Kong, Hong Kong, SAR, People's Republic of China.

Received: 26 February 2018 Accepted: 13 August 2018

Published online: 28 August 2018

\section{References}

1. Baldovino S, Moliner AM, Taruscio D, Daina E, Roccatello D. Rare diseases in Europe: from a wide to a local perspective. Isr Med Assoc J. 2016;18:359-63.

2. Petr Šimerka. Council Recommendation of 8 June 2009 on European action in the field of rare diseases. Off J Eur Union. 2009;C151:7-10.

3. Elliott E, Zurynski Y. Rare diseases are a 'common' problem for clinicians. Aust Fam Physician. 2015:44:630-3.

4. EURORDIS: Survey of the delay in diagnosis for 8 rare diseases in Europe ('EurordisCare2'). 2007.

5. Molster C, Urwin D, Di Pietro L, Fookes M, Petrie D, van der Laan S, Dawkins $\mathrm{H}$. Survey of healthcare experiences of Australian adults living with rare diseases. Orphanet J Rare Dis. 2016;11:30.

6. Richter T, Nestler-Parr S, Babela R, Khan ZM, Tesoro T, Molsen E, Hughes DA, International Society for $\mathrm{P}$, outcomes research rare disease special interest G. Rare disease terminology and definitions-a systematic global review: report of the ISPOR rare disease special interest group. Value Health. 2015; 18:906-14.

7. Boycott KM, Rath A, Chong JX, Hartley T, Alkuraya FS, Baynam G, Brookes AJ, Brudno M, Carracedo A, den Dunnen JT, et al. International cooperation to enable the diagnosis of all rare genetic diseases. Am J Hum Genet. 2017; 100:695-705.

8. Lin JD, Lin LP, Hung WJ. Reported numbers of patients with rare diseases based on ten-year longitudinal national disability registries in Taiwan. Res Dev Disabil. 2013;34:133-8.

9. Mazzucato M, Visona Dalla Pozza L, Manea S, Minichiello C, Facchin P. A population-based registry as a source of health indicators for rare diseases: the ten-year experience of the Veneto Region's rare diseases registry. Orphanet J Rare Dis. 2014;9:37.

10. Rath A, Olry A, Dhombres F, Brandt MM, Urbero B, Ayme S. Representation of rare diseases in health information systems: the Orphanet approach to serve a wide range of end users. Hum Mutat. 2012;33:803-8.

11. Walker CE, Mahede T, Davis G, Miller LJ, Girschik J, Brameld K, Sun W, Rath A, Ayme S, Zubrick SR, et al. The collective impact of rare diseases in Western Australia: an estimate using a population-based cohort. Genet Med 2017;19:546-52.

12. Census and Statistics Department. The Government of the Hong Kong Special Administrative Region: Hong Kong Annual Digest of Statistics, 2017 edition. 2017.

13. Hospital Authority HK: Hospital authority statistical report 2015-2016. 2016.

14. The Government of the Hong Kong Special Administrative Region. Editorial Guide on Hong Kong Clinical Terminology Table - Problem \& Procedure. Version 1.1. 2017.

15. Mok CC. Epidemiology and survival of systemic lupus erythematosus in Hong Kong Chinese. Lupus. 2011;20:767-71.

16. Yim WW, Graham CA, Rainer TH. A comparison of emergency department utilization by elderly and younger adult patients presenting to three hospitals in Hong Kong. Int J Emerg Med. 2009;2:19-24.

17. DiFazio RL, Vessey JA. Non-medical out-of-pocket expenses incurred by families during their child's hospitalization. J Child Health Care. 2013;17:230-41.

18. Chiu SS, Lau YL, Chan KH, Wong WH, Peiris JS. Influenza-related hospitalizations among children in Hong Kong. N Engl J Med. 2002; 347:2097-103. 